\title{
Studies of the ABJM theory in a formulation with manifest SU(4) R-symmetry
}

\author{
Miguel A. Bandres, Arthur E. Lipstein and John H. Schwarz \\ California Institute of Technology, \\ Pasadena, CA 91125, U.S.A. \\ E-mail: bandres@caltech.edu, arthur@caltech.edu, jhs@theory.caltech.edu
}

\begin{abstract}
We examine the three-dimensional $\mathcal{N}=6$ superconformal Chern-Simons theory with $\mathrm{U}(N) \times \mathrm{U}(N)$ gauge symmetry, which was recently constructed by Aharony, Bergman, Jafferis, and Maldacena (ABJM). Using a formulation with manifest SU(4) Rsymmetry and no auxiliary fields, we verify in complete detail both the Poincaré supersymmetry and the conformal supersymmetry of the action. Together, these imply the complete $O S p(6 \mid 4)$ superconformal symmetry of the theory. The potential, which is sixth order in scalar fields, is recast as a sum of squares.
\end{abstract}

KEYwords: Conformal Field Models in String Theory, AdS-CFT Correspondence, M-Theory. 


\section{Contents}

1. Introduction 1

2. The $\mathrm{U}(1) \times \mathrm{U}(1)$ theory 3

3. The $\mathrm{U}(N) \times \mathrm{U}(N)$ theory 5

4. Conclusion 7

A. Verification of superconformal symmetry 8

\section{Introduction}

This paper examines a new class of superconformal field theories in three dimensions that was recently discovered by Aharony, Bergman, Jafferis, and Maldacena (ABJM) [1]. These theories are superconformal Chern-Simons gauge theories with $\mathcal{N}=6$ supersymmetry. When the gauge group is chosen to be $\mathrm{U}(N) \times \mathrm{U}(N)$ and the Chern-Simons level is $k$, these theories are conjectured to be dual to M-theory on $A d S_{4} \times S^{7} / \mathbb{Z}_{k}$ with $N$ units of flux. More precisely, this is the appropriate dual description for $N^{1 / 5} \gg k$. In the opposite limit, $N^{1 / 5} \ll k \ll N$, a dual description in terms of type IIA string theory on $A d S_{4} \times \mathbb{C} P^{3}$ is more appropriate. A large- $N$ expansion for fixed 't Hooft parameter $\lambda=N / k$ can be defined. These developments raise the hope that this duality can be analyzed in the same level of detail as has been done for the duality between $\mathcal{N}=4$ super Yang-Mills theory with a $\mathrm{U}(N)$ gauge group in four dimensions and type IIB superstring theory on $A d S_{5} \times S^{5}$ with $N$ units of flux.

Even though the ABJM paper is very recent, quite a few papers have already appeared that examine various of its properties as well as possible generalizations. Among the first are [2]-15. New superconformal Chern-Simons theories with $\mathcal{N}=5$ supersymmetry have been constructed in [11]. (This paper also does many other things.) Certain of these $\mathcal{N}=5$ theories should be dual to the $D_{k+2}$ orbifolds described in [12]. Also, in a very interesting recent paper [13], Bagger and Lambert show that the ABJM theories correspond to a class of 3-algebras in which the bracket $\left[T^{a}, T^{b}, T^{c}\right]$ is no longer antisymmetric in all three indices. The actions and supersymmetry transformations that are derived in [11, 13] appear to be equivalent to the actions and supersymmetry transformations that are obtained in this paper (without reference to 3 -algebras).

The three-dimensional superconformal field theories of coincident M2-branes were initially defined as infrared fixed points of super Yang-Mills theories, i.e., as limits of the form $g_{\mathrm{YM}} \rightarrow \infty$. In 16] it was proposed that these fixed points could be reformulated 
in a more useful dual formulation analogous to a Seiberg dual. It was suggested that the theory would be a gauge theory in which the gauge fields couple to dimension- $1 / 2$ scalar and dimension- 1 spinor fields. Since all terms should be dimension 3 , there should be no dimension- $4 F^{2}$ kinetic terms, but dimension-3 Chern-Simons terms would be allowed. An approach to constructing such theories based on considering multiple M2-branes ending on an M5-brane was proposed in 17. Several years later, a specific example of such a superconformal Chern-Simons theory with maximal $(\mathcal{N}=8)$ supersymmetry was constructed by Bagger and Lambert [18-20] and by Gustavsson [21, 22]. This theory is parity conserving and has $\mathrm{SO}(4)=\mathrm{SU}(2) \times \mathrm{SU}(2)$ gauge symmetry 23 , 24]. The scalars and spinors are 4 -vectors of $\mathrm{SO}(4)$, or (equivalently) bifundamentals of $\mathrm{SU}(2) \times \mathrm{SU}(2)$.

The BLG theory was conjectured [23] and proved [25, 26] to be the unique theory of this type with maximal supersymmetry. (Generalizations based on Lorentzian 3-algebras [2729] turned out to be equivalent to the original super Yang-Mills theories once the ghosts were eliminated [30-32].) This left the possibility of considering theories with reduced supersymmetry. A large class of superconformal Chern-Simons theories with $\mathcal{N}=4$ supersymmetry was constructed by Gaiotto and Witten 33. This was generalized to include twisted hypermultiplets in [11, 34]. This generalization includes the Bagger-Lambert theory as a special case. Moreover, all the ABJM theories turn out to be special cases of the generalized Gaiotto-Witten theories in which the supersymmetry is enhanced to $\mathcal{N}=6$. The dual M-theory picture requires that for levels $k=1,2$ the ABJM theories should have $\mathcal{N}=8$ supersymmetry. However, this has not yet been demonstrated explicitly.

The purpose of this paper is to recast the ABJM theory in a form for which the $\mathrm{SU}(4) \mathrm{R}$-symmetry of the action and the supersymmetry transformations is manifest and to use this form to study some of its properties. The existence of such formulas is a consequence of what was found in [1]. We also verify the conformal supersymmetry of the action, which is not a logical consequence of previous results. Since this symmetry is a necessary requirement for the validity of the proposed duality, its verification can be viewed as an important and nontrivial test of the duality. We also recast the potential, which is sixth order in the scalar fields, in a new form. ${ }^{1}$ This new form should be useful for studying the moduli space of supersymmetric vacua of the theory, as well as the vacuum structure of various deformations of the ABJM theory. Although we discuss the gauge group $\mathrm{U}(N) \times \mathrm{U}(N)$, all of our analysis also holds for the straightforward generalization to $\mathrm{U}(M) \times \mathrm{U}(N)$.

Some of our results are new and others confirm results that have been obtained previously. The ABJM theories were formulated in [1] using auxiliary fields associated with $\mathcal{N}=2$ superfields. In this formulation only an $\mathrm{SU}(2) \times \mathrm{SU}(2)$ subgroup of the $\mathrm{SU}(4) \mathrm{R}-$ symmetry is manifest, though the full SU(4) symmetry has been deduced. In addition, [1] deduced a manifestly SU(4) invariant form of the scalar field potential, which is sixth order in the scalar fields. The quartic interaction terms that have two scalar and two spinor fields were also recast in an SU(4) covariant form in [2]. Our results are in agreement with both of these.

\footnotetext{
${ }^{1} \mathrm{~A}$ similar formula also appears in $\sqrt{13}$.
} 


\section{The $\mathrm{U}(1) \times \mathrm{U}(1)$ theory}

The field content of ABJM theories consists of scalars, spinors, and gauge fields. The $\mathrm{U}(1) \times \mathrm{U}(1)$ theory has fewer indices to keep track of, and it is quite a bit simpler, than the full $\mathrm{U}(N) \times \mathrm{U}(N)$ theory; so it is a good place to start.

There are four complex scalars $X_{A}$ and their adjoints $X^{A}$. (We choose not to use adjoint or complex conjugation symbols to keep the notation from becoming too cumbersome.) A lower index labels the 4 representation of the global SU(4) R-symmetry and an upper index labels the complex-conjugate $\overline{4}$ representation.

Similarly, the fermi fields are $\Psi^{A}$ and $\Psi_{A}$. These are also two-component spinors, though that index is not displayed. As usual, the notation $\bar{\Psi}^{A}$ or $\bar{\Psi}_{A}$ implies transposing the spinor index and right multiplication by $\gamma^{0}$. Note, however, that for our definition there is no additional complex conjugation, so in all cases a lower index indicates a 4 and an upper index indicates a $\overline{\mathbf{4}}$. With these conventions various identities that hold for Majorana spinors can be used for these spinors, as well, even though they are complex (Dirac). For example, $\bar{\Psi}^{A} \Psi_{B}=\bar{\Psi}_{B} \Psi^{A}$. The $2 \times 2$ Dirac matrices satisfy $\left\{\gamma^{\mu}, \gamma^{\nu}\right\}=2 \eta^{\mu \nu}$. The index $\mu=0,1,2$ is a 3 -dimensional Lorentz index, and the signature is $(-,+,+)$. It is convenient to use a Majorana representation, which implies that $\gamma^{\mu}$ is real. We also choose a representation for which $\gamma^{\mu \nu \lambda}=\varepsilon^{\mu \nu \lambda}$. In particular, this means that $\gamma^{0} \gamma^{1} \gamma^{2}=1$. For example, one could choose $\gamma^{0}=i \sigma^{2}, \gamma^{1}=\sigma^{1}$, and $\gamma^{2}=\sigma^{3}$.

The $\mathrm{U}(1)$ gauge fields are denoted $A_{\mu}$ and $\hat{A}_{\mu}$. The fields $X_{A}$ and $\Psi^{A}$ have $\mathrm{U}(1)$ charges $(+,-)$, while their adjoints have charges $(-,+)$. Thus, for example,

$$
D_{\mu} X_{A}=\partial_{\mu} X_{A}+i\left(A_{\mu}-\hat{A}_{\mu}\right) X_{A}
$$

and

$$
D_{\mu} X^{A}=\partial_{\mu} X^{A}-i\left(A_{\mu}-\hat{A}_{\mu}\right) X^{A} .
$$

We choose to normalize fields so that the level- $k$ Lagrangian is $k$ times the level-1 Lagrangian. With this convention, the $N=1$ action is

$$
S=\frac{k}{2 \pi} \int d^{3} x\left(-D^{\mu} X^{A} D_{\mu} X_{A}+i \bar{\Psi}_{A} \gamma^{\mu} D_{\mu} \Psi^{A}+\frac{1}{2} \varepsilon^{\mu \nu \lambda}\left(A_{\mu} \partial_{\nu} A_{\lambda}-\hat{A}_{\mu} \partial_{\nu} \hat{A}_{\lambda}\right)\right) .
$$

The claim is that this action describes an $\mathcal{N}=6$ superconformal theory with $O S p(6 \mid 4)$ superconformal symmetry. The R-symmetry is $\operatorname{Spin}(6)=\mathrm{SU}(4)$ and the conformal symmetry is $\operatorname{Sp}(4)=\operatorname{Spin}(3,2)$. The supercharges transform as the 6 representation of SU(4). Both the Poincaré and conformal supercharges are 6-vectors. Each accounts for 12 of the 24 fermionic generators of the superconformal algebra.

The antisymmetric product of two $4 \mathrm{~s}$ gives a 6 . The invariant tensor (or ClebschGordan coefficients) describing this is denoted $\Gamma_{A B}^{I}=-\Gamma_{B A}^{I}$, since these can be interpreted as six matrices satisfying a Clifford algebra. More precisely, if one also defines $\tilde{\Gamma}^{I}=\left(\Gamma^{I}\right)^{\dagger}$, or in components

$$
\tilde{\Gamma}^{I A B}=\frac{1}{2} \varepsilon^{A B C D} \Gamma_{C D}^{I}=-\left(\Gamma_{A B}^{I}\right)^{*},
$$


then $^{2}$

$$
\Gamma^{I} \tilde{\Gamma}^{J}+\Gamma^{J} \tilde{\Gamma}^{I}=2 \delta^{I J} .
$$

Note that $\gamma^{\mu}$ are $2 \times 2$ matrices and $\Gamma^{I}$ are $4 \times 4$ matrices. They act on different vector spaces, and therefore they trivially commute with one another.

The supersymmetry transformations of the matter fields are

$$
\begin{aligned}
\delta X_{A} & =i \Gamma_{A B}^{I} \bar{\Psi}^{B} \varepsilon^{I} \\
\delta \Psi_{A} & =\Gamma_{A B}^{I} \gamma^{\mu} \varepsilon^{I} D_{\mu} X^{B}
\end{aligned}
$$

and their adjoints, which are

$$
\begin{aligned}
\delta X^{A} & =-i \tilde{\Gamma}^{I A B} \bar{\Psi}_{B} \varepsilon^{I} \\
\delta \Psi^{A} & =-\tilde{\Gamma}^{I A B} \gamma^{\mu} \varepsilon^{I} D_{\mu} X_{B} .
\end{aligned}
$$

For the gauge fields we have

$$
\delta A_{\mu}=\delta \hat{A}_{\mu}=-\Gamma_{A B}^{I} \bar{\Psi}^{A} \gamma_{\mu} \varepsilon^{I} X^{B}-\tilde{\Gamma}^{I A B} \bar{\Psi}_{A} \gamma_{\mu} \varepsilon^{I} X_{B}
$$

The verification that these leave the action invariant is given in the appendix.

Note that the covariant derivatives only involve $A_{-}$, where

$$
A_{ \pm}=A \pm \hat{A}
$$

Therefore, let us rewrite the Chern-Simons terms using 35]

$$
\int(A \wedge d A-\hat{A} \wedge d \hat{A})=\int A_{+} \wedge d A_{-}=\int A_{-} \wedge d A_{+} .
$$

Since this is the only appearance of $A_{+}$in the action, it can be integrated out to give the delta functional constraint

$$
F_{-}=d A_{-}=0
$$

The $A_{-}$equation of motion, on the other hand, just identifies $F_{+}$with the dual of the charge current. Since the kinetic terms are defined with a flat connection $A_{-}$, this is just a free theory when the topology is trivial, which is the case for $k=1$. Then this theory has $\mathcal{N}=8$ superconformal symmetry.

ABJM proposes to treat $F_{+}$as an independent variable and to add a Lagrange multiplier term to ensure that $F_{+}$is a curl

$$
S_{\tau}=\frac{1}{4 \pi} \int \tau \varepsilon^{\mu \nu \lambda} \partial_{\mu} F_{+\nu \lambda} d^{3} x
$$

Then the quantization condition on $F_{+}$requires that $\tau$ has period $2 \pi$. They then explain that after gauge fixing $\tau=0$ one is left with a residual $\mathbb{Z}_{k}$ gauge symmetry under which $X^{A} \rightarrow \exp (2 \pi i / k) X^{A}$ and similarly for $\Psi_{A}$. Thus one is left with a sigma model on $\mathbb{C}^{4} / \mathbb{Z}_{k}$.

\footnotetext{
${ }^{2}$ An explicit realization in terms of Pauli matrices is given by $\Gamma^{1}=i \sigma_{2} \otimes 1, \Gamma^{2}=\sigma_{2} \otimes \sigma_{1}, \Gamma^{3}=\sigma_{2} \otimes \sigma_{3}$, $\Gamma^{4}=1 \otimes \sigma_{2}, \Gamma^{5}=i \sigma_{1} \otimes \sigma_{2}, \Gamma^{6}=i \sigma_{3} \otimes \sigma_{2}$.
} 
This breaks the supersymmetry from $\mathcal{N}=8$ to $\mathcal{N}=6$ for $k>2$. The reason for this is that the 8-component $\operatorname{Spin}(8)$ supercharge decomposes with respect to the $\mathrm{SU}(4) \times \mathrm{U}(1)$ subgroup as $6_{0}+1_{2}+1_{-2}$. Because of their $\mathrm{U}(1)$ charges, the singlets transform under a $\mathbb{Z}_{k}$ transformation as $Q \rightarrow \exp ( \pm 4 \pi i / k) Q$. Therefore two of the supersymmetries are broken for $k>2$.

This analysis of the $\mathrm{U}(1)$ factors continues to apply in the $\mathrm{U}(N) \times \mathrm{U}(N)$ theories with $N>1$. The Bagger-Lambert theory corresponds to the gauge group $\mathrm{SU}(2) \times \mathrm{SU}(2)$. Since it has no $\mathrm{U}(1)$ factors, no discrete $Z_{k}$ gauge symmetry arises, and this theory has $\mathcal{N}=8$ superconformal symmetry for all values of $k$. So, it is different from the $\mathrm{U}(2) \times \mathrm{U}(2)$ ABJM theory, and its interpretation in terms of branes or geometry (see [36, 37]) must also be different.

\section{The $\mathrm{U}(N) \times \mathrm{U}(N)$ theory}

The field content of the $\mathrm{U}(N) \times \mathrm{U}(N)$ ABJM theory consists of four $N \times N$ matrices of complex scalars $\left(X_{A}\right)^{a}{ }_{\hat{a}}$ and their adjoints $\left(X^{A}\right)^{\hat{a}}{ }_{a}$. These transform as $(\overline{\mathbf{N}}, \mathbf{N})$ and $(\mathbf{N}, \overline{\mathbf{N}})$ representations of the gauge group, respectively. Similarly, the spinor fields are matrices $\left(\Psi^{A}\right)^{a}{ }_{\hat{a}}$ and their adjoints $\left(\Psi_{A}\right)^{\hat{a}}{ }_{a}$. The $\mathrm{U}(N)$ gauge fields are hermitian matrices $A^{a}{ }_{b}$ and $\hat{A}_{\hat{b}}^{\hat{a}_{\hat{~}}}$. In matrix notation, the covariant derivatives are

$$
D_{\mu} X_{A}=\partial_{\mu} X_{A}+i\left(A_{\mu} X_{A}-X_{A} \hat{A}_{\mu}\right)
$$

and

$$
D_{\mu} X^{A}=\partial_{\mu} X^{A}+i\left(\hat{A}_{\mu} X^{A}-X^{A} A_{\mu}\right)
$$

with similar formulas for the spinors. Infinitesimal gauge transformations are given by

$$
\begin{aligned}
\delta A_{\mu} & =D_{\mu} \Lambda=\partial_{\mu} \Lambda+i\left[A_{\mu}, \Lambda\right] \\
\delta \hat{A}_{\mu} & =D_{\mu} \hat{\Lambda}=\partial_{\mu} \hat{\Lambda}+i\left[\hat{A}_{\mu}, \hat{\Lambda}\right] \\
\delta X_{A} & =-i \Lambda X_{A}+i X_{A} \hat{\Lambda}
\end{aligned}
$$

The action consists of terms that are straightforward generalizations of those of the $\mathrm{U}(1) \times \mathrm{U}(1)$ theory, as well as new interaction terms that vanish for $N=1$. The kinetic and Chern-Simons terms are

$$
S_{\text {kin }}=\frac{k}{2 \pi} \int d^{3} x \operatorname{tr}\left(-D^{\mu} X^{A} D_{\mu} X_{A}+i \bar{\Psi}_{A} \gamma^{\mu} D_{\mu} \Psi^{A}\right) .
$$

and

$$
S_{\mathrm{CS}}=\frac{k}{2 \pi} \int d^{3} x \varepsilon^{\mu \nu \lambda} \operatorname{tr}\left(\frac{1}{2} A_{\mu} \partial_{\nu} A_{\lambda}+\frac{i}{3} A_{\mu} A_{\nu} A_{\lambda}-\frac{1}{2} \hat{A}_{\mu} \partial_{\nu} \hat{A}_{\lambda}-\frac{i}{3} \hat{A}_{\mu} \hat{A}_{\nu} \hat{A}_{\lambda}\right) .
$$

Additional interaction terms of the schematic form $X^{2} \Psi^{2}$ and $X^{6}$ remain to be determined. These terms are not required to deduce the equations of motion of the gauge fields, which are

$$
J^{\mu}=\frac{1}{2} \varepsilon^{\mu \nu \lambda} F_{\nu \lambda} \quad \hat{J}^{\mu}=-\frac{1}{2} \varepsilon^{\mu \nu \lambda} \hat{F}_{\nu \lambda}
$$


where

$$
J^{\mu}=i X_{A} D^{\mu} X^{A}-i D^{\mu} X_{A} X^{A}-\bar{\Psi}^{A} \gamma^{\mu} \Psi_{A}
$$

and

$$
\hat{J}^{\mu}=i X^{A} D^{\mu} X_{A}-i D^{\mu} X^{A} X_{A}-\bar{\Psi}_{A} \gamma^{\mu} \Psi^{A} .
$$

Note that in the special case of $\mathrm{U}(1) \times \mathrm{U}(1)$ one has $J^{\mu}=-\hat{J}^{\mu}$, and hence the equations of motion imply $F_{\mu \nu}=\hat{F}_{\mu \nu}$.

In matrix notation, the supersymmetry transformations of the matter fields are

$$
\delta X_{A}=i \Gamma_{A B}^{I} \bar{\varepsilon}^{I} \Psi^{B}
$$

and

$$
\delta \bar{\Psi}_{A}=-\Gamma_{A B}^{I} \bar{\varepsilon}^{I} \gamma^{\mu} D_{\mu} X^{B}+\delta_{3} \bar{\Psi}_{A}
$$

or equivalently

$$
\delta \Psi_{A}=\Gamma_{A B}^{I} \gamma^{\mu} \varepsilon^{I} D_{\mu} X^{B}+\delta_{3} \Psi_{A} .
$$

and their adjoints, which are

$$
\delta X^{A}=-i \tilde{\Gamma}^{I A B} \bar{\Psi}_{B} \varepsilon^{I}
$$

and

$$
\delta \Psi^{A}=-\tilde{\Gamma}^{I A B} \gamma^{\mu} \varepsilon^{I} D_{\mu} X_{B}+\delta_{3} \Psi^{A} .
$$

or equivalently

$$
\delta \bar{\Psi}^{A}=\tilde{\Gamma}^{I A B} \bar{\varepsilon}^{I} \gamma^{\mu} D_{\mu} X_{B}+\delta_{3} \bar{\Psi}^{A} .
$$

The terms denoted $\delta_{3}$ are cubic in $X$ and are given below. The supersymmetry transformations of the gauge fields are

$$
\begin{aligned}
& \delta A_{\mu}=\Gamma_{A B}^{I} \bar{\varepsilon}^{I} \gamma_{\mu} \Psi^{A} X^{B}-\tilde{\Gamma}^{I A B} X_{B} \bar{\Psi}_{A} \gamma_{\mu} \varepsilon^{I} \\
& \delta \hat{A}_{\mu}=\Gamma_{A B}^{I} X^{B} \bar{\varepsilon}^{I} \gamma_{\mu} \Psi^{A}-\tilde{\Gamma}^{I A B} \bar{\Psi}_{A} \gamma_{\mu} \varepsilon^{I} X_{B} .
\end{aligned}
$$

Note that $\delta A_{\mu} \neq \delta \hat{A}_{\mu}$ for $N>1$. They are matrices in different spaces.

In the appendix we show that supersymmetry requires the choice

$$
\delta_{3} \Psi^{A}=N^{I A} \varepsilon^{I} \quad \delta_{3} \Psi_{A}=N_{A}^{I} \varepsilon^{I},
$$

where

$$
N^{I A}=\tilde{\Gamma}^{I A B}\left(X_{C} X^{C} X_{B}-X_{B} X^{C} X_{C}\right)-2 \tilde{\Gamma}^{I B C} X_{B} X^{A} X_{C} .
$$

and

$$
N_{A}^{I}=\left(N^{I A}\right)^{\dagger}=\Gamma_{A B}^{I}\left(X^{C} X_{C} X^{B}-X^{B} X_{C} X^{C}\right)-2 \Gamma_{B C}^{I} X^{B} X_{A} X^{C} .
$$

Note that these expressions vanish when the matrices $X^{A}$ (and their adjoints $X_{A}$ ) are diagonal.

All the possible structures for the $\Psi^{2} X^{2}$ terms are

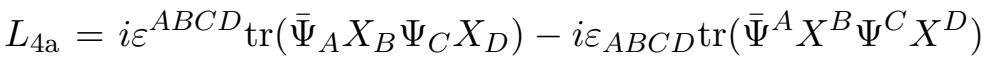

$$
\begin{aligned}
& L_{4 \mathrm{~b}}=i \operatorname{tr}\left(\bar{\Psi}^{A} \Psi_{A} X_{B} X^{B}\right)-i \operatorname{tr}\left(\bar{\Psi}_{A} \Psi^{A} X^{B} X_{B}\right) \\
& L_{4 \mathrm{c}}=2 i \operatorname{tr}\left(\bar{\Psi}_{A} \Psi^{B} X^{A} X_{B}\right)-2 i \operatorname{tr}\left(\bar{\Psi}^{B} \Psi_{A} X_{B} X^{A}\right)
\end{aligned}
$$


The coefficients are chosen so that $L_{4}=L_{4 \mathrm{a}}+L_{4 \mathrm{~b}}+L_{4 \mathrm{c}}$ is the correct result required by supersymmetry, as is demonstrated in the appendix.

The lagrangian also contains a term $L_{6}=-V$ that is sixth order in the scalar fields. The scalar potential $V$ is expected to be nonnegative and to vanish for a supersymmetric vacuum. An SU(4) covariant formula for $V$ in terms of the fields $X^{A}$ and $X_{A}$ has been given in [1, 2]

$$
\begin{aligned}
V=-\frac{1}{3} \operatorname{tr}\left[X^{A} X_{A} X^{B} X_{B} X^{C} X_{C}+X_{A} X^{A} X_{B} X^{B} X_{C} X^{C}\right. \\
\left.+4 X_{A} X^{B} X_{C} X^{A} X_{B} X^{C}-6 X^{A} X_{B} X^{B} X_{A} X^{C} X_{C}\right]
\end{aligned}
$$

a result that we confirm in the appendix.

This formula for $V$ is not expressed as a sum of squares, which makes it inconvenient for determining the extrema. For a supersymmetric vacuum, $\delta \Psi^{A}=\delta \Psi_{A}=0$. In particular, for a solution in which the scalar fields $X^{A}$ and $X_{A}$ are constant, and the gauge fields vanish, the variations $\delta_{3} \Psi^{A}$ and $\delta_{3} \Psi_{A}$ should vanish. This implies that $N^{I A}=0$ and $N_{A}^{I}=\left(N^{I A}\right)^{\dagger}=0$. The way to ensure these requirements, as well as manifest $\mathrm{SU}(4)$ symmetry, is for the potential to take the form

$$
V=\frac{1}{6} \operatorname{tr}\left(N^{I A} N_{A}^{I}\right)
$$

The definitions of $N^{I A}$ and $N_{A}^{I}$ are given in eqs. 3.20) and (3.21). It is straightforward to verify the equivalence of eqs. (3.25) and (3.26) for this choice of the coefficient by using the key identity

$$
\Gamma_{A B}^{I} \tilde{\Gamma}^{I C D}=-2 \delta_{A B}^{C D}
$$

The indicated relationship between the potential and $\delta_{3} \Psi$ in eq. (3.26) should be quite general in theories of this type. As has already been noted, $N^{I A}$ and $N_{A}^{I}$ vanish when the scalar fields are diagonal matrices. To get the expected moduli space, these should be the only choices for which they vanish (modulo gauge transformations).

\section{Conclusion}

The study of ABJM theories has become a hot topic. The technology that has been developed in the study of the duality between four-dimensional superconformal gauge theories and $A d S_{5}$ vacua of type IIB superstring theory can now be adapted to a new setting. It should now be possible to study the duality between three-dimensional superconformal Chern-Simon theories and $A d S_{4}$ vacua of type IIA superstring theory and M-theory. A great deal should be learned in the process, and there may even be applications to other areas of physics.

Our contribution to this subject is modest: We have verified the Poincaré supersymmetries of the ABJM theory in a formalism with manifest SU(4) symmetry. The action that we obtained agrees with results given in [1, 2, 13]. We have also verified by explicit calculation that this action has the conformal supersymmetries that are required by the proposed duality. Since this is not implied by any previous calculations, it is an important 
(and nontrivial) test of the duality. Taken together with the Poincaré supersymmetries, this implies the full $O S p(6 \mid 4)$ superconformal symmetry of the action. We have also recast the sextic potential as a sum of squares in eq. (3.26), a form that should prove useful in future studies.

\section{Acknowledgments}

This work was supported in part by the U.S. Dept. of Energy under Grant No. DE-FG0392-ER40701. MAB acknowledges support from the Secretaría de Educación Publica de México. JHS wishes to acknowledge the hospitality of the Aspen Center for Physics. He also acknowledges helpful discussions with Igor Klebanov.

\section{A. Verification of superconformal symmetry}

The $\mathbf{U}(\mathbf{1}) \times \mathbf{U}(\mathbf{1})$ theory. Let us check the supersymmetry of the $\mathrm{U}(1) \times \mathrm{U}(1)$ theory. We only analyze half of the terms, since the other half are just their adjoints. Omitting the factor of $k / 2 \pi$, the variation of the Lagrangian contains (dropping total derivatives)

$$
\Delta_{1}=-D^{\mu} X^{A} D_{\mu} \delta X_{A}=i D^{2} X^{A} \bar{\varepsilon}^{I} \Gamma_{A B}^{I} \Psi^{B}
$$

and

$$
\begin{aligned}
\Delta_{2} & =i \delta \bar{\Psi}_{A} \gamma \cdot D \Psi^{A} \\
& =-i \Gamma_{A B}^{I} \bar{\varepsilon}^{I} \gamma \cdot D X^{B} \gamma \cdot D \Psi^{A} \\
& =i \Gamma_{A B}^{I} \bar{\varepsilon}^{I} D^{2} X^{B} \Psi^{A}-\frac{1}{2} \Gamma_{A B}^{I} \bar{\varepsilon}^{I} \gamma^{\rho \mu}\left(F_{\rho \mu}-\hat{F}_{\rho \mu}\right) X^{B} \Psi^{A}
\end{aligned}
$$

Note that the gauge fields only appear in the covariant derivatives in the combination $A-\hat{A}$, which has a vanishing supersymmetry variation. The variation of the Chern-Simons term, using the first term in eq. (2.10), contributes

$$
\Delta_{3}=\frac{1}{2} \varepsilon^{\mu \nu \lambda} \bar{\varepsilon}^{I} \gamma_{\mu} \Psi^{A} \Gamma_{A B}^{I} X^{B}\left(F_{\nu \lambda}-\hat{F}_{\nu \lambda}\right) .
$$

Using $\varepsilon^{\mu \nu \lambda} \gamma_{\mu}=\gamma^{\nu \lambda}$, we see that $\Delta_{1}+\Delta_{2}+\Delta_{3}=0$. The other half of the terms in the variation of the action, which are the adjoints of the ones considered here, cancel in the same way. The conserved supersymmetry current can be computed by the standard Noether procedure. This gives (aside from an arbitrary normalization)

$$
Q_{\mu}^{I}=\Gamma_{A B}^{I} \gamma \cdot D X^{A} \gamma_{\mu} \Psi^{B}-\tilde{\Gamma}^{I A B} \gamma \cdot D X_{A} \gamma_{\mu} \Psi_{B}
$$

One can check this result by computing the divergence. This vanishes as a consequence of the equations of motion $\gamma \cdot D \Psi^{B}=0, D \cdot D X^{A}=0$, and $F_{\mu \nu}-\hat{F}_{\mu \nu}=0$.

Let us now explore the conformal supersymmetry, with an infinitesimal spinor parameter $\eta^{I}$, using the method explained in [23]. As a first try, consider replacing $\varepsilon^{I}$ by $\gamma \cdot x \eta^{I}$ in the preceding equations, since this has the correct dimensions. Using $\partial_{\mu} \varepsilon(x)=\gamma_{\mu} \eta$ and 
$\gamma^{\mu} \gamma^{\rho} \gamma_{\mu}=-\gamma^{\rho}$, this gives a variation of the action that almost cancels, except for a couple of terms. These remaining terms can be canceled by including an additional variation of the spinor fields. It has the form

$$
\delta^{\prime} \Psi^{A}=-\tilde{\Gamma}^{I A B} \eta^{I} X_{B} \quad \delta^{\prime} \Psi_{A}=\Gamma_{A B}^{I} \eta^{I} X^{B} .
$$

Correspondingly, the conserved superconformal current is

$$
S_{\mu}^{I}=\gamma \cdot x Q_{\mu}^{I}+\Gamma_{A B}^{I} X^{A} \gamma_{\mu} \Psi^{B}-\tilde{\Gamma}^{I A B} X_{A} \gamma_{\mu} \Psi_{B} .
$$

As a check, one can compute the divergence using the conservation of $Q_{\mu}^{I}$ and the spinor field equation of motion

$$
\partial^{\mu} S_{\mu}^{I}=\gamma^{\mu} Q_{\mu}^{I}+\Gamma_{A B}^{I} \gamma \cdot D X^{A} \Psi^{B}-\tilde{\Gamma}^{I A B} \gamma \cdot D X_{A} \Psi_{B}=0 .
$$

The various bosonic $O S p(6 \mid 4)$ symmetry transformations are obtained by commuting $\varepsilon$ and $\eta$ transformations. Of these only the conformal transformation, obtained as the commutator of two $\eta$ transformations, is not a manifest symmetry of the action. It is often true that scale invariance implies conformal symmetry. However, this is not a general theorem, so it is a good idea to check the conformal symmetry (or the conformal supersymmetry) explicitly.

The $\mathbf{U}(\boldsymbol{N}) \times \mathbf{U}(\boldsymbol{N})$ theory. Let us now examine the supersymmetry of the $\mathrm{U}(N) \times \mathrm{U}(N)$ theory. Some of the terms are simple generalizations of those examined in the $N=1$ case and will not be described here. Rather, we focus on those that only arise for $N>1$. We will first determine the quartic $\Psi^{2} X^{2}$ term (called $L_{4}$ ) in the action by requiring that the variation of its $X$ fields cancels the terms that arise from varying the gauge fields in the spinor kinetic term. Since these terms are cubic in $\Psi$, various Fierz identities are required. The second step is to determine the variation $\delta_{3} \Psi$ by requiring that this variation of the spinor kinetic term cancels against the lowest-order variation of the $\Psi$ fields in $L_{4}$ and the variation of the gauge fields in the scalar kinetic term. The third and final step is to determine $L_{6}$ by arranging that its variation cancels against the $\delta_{3} \Psi$ variation of $L_{4}$. After this has been completed, we verify the conformal supersymmetry.

Determination of $\boldsymbol{L}_{4}$. A useful identity involving four two-component Majorana spinors, obtained by a Fierz transformation, is

$$
\bar{\psi}_{1} \gamma_{\mu} \psi_{2} \bar{\psi}_{3} \gamma^{\mu} \varepsilon=-2 \bar{\varepsilon} \psi_{1} \bar{\psi}_{2} \psi_{3}-\bar{\psi}_{1} \psi_{2} \bar{\varepsilon} \psi_{3} .
$$

Juggling the indices this can be recast in the form

$$
\bar{\varepsilon} \gamma_{\mu} \psi_{1} \bar{\psi}_{2} \gamma^{\mu} \psi_{3}=-2 \bar{\psi}_{1} \psi_{2} \bar{\varepsilon} \psi_{3}-\bar{\varepsilon} \psi_{1} \bar{\psi}_{2} \psi_{3} .
$$

These will be useful for eliminating Dirac matrices from equations that arise later. As written, these relations preserve the 123 sequence of the spinors, which is convenient if they are matrices that are to be multiplied. However, the right-hand sides can be rewritten in other ways without Dirac matrices using the relation

$$
\psi_{1} \bar{\psi}_{2} \psi_{3}+\psi_{2} \bar{\psi}_{3} \psi_{1}+\psi_{3} \bar{\psi}_{1} \psi_{2}=0 \text {. }
$$


This equation will also be useful.

Varying the gauge fields in the spinor kinetic term of the $\mathrm{U}(N) \times \mathrm{U}(N)$ theory (dropping a factor of $k / 2 \pi$ ) gives

$$
\operatorname{tr}\left(\bar{\Psi}_{A} \gamma^{\mu}\left(-\delta A_{\mu} \Psi^{A}+\Psi^{A} \delta \hat{A}_{\mu}\right)\right)
$$

Keeping only the terms with two superscripts on spinor fields, since the other terms are just their adjoints, leaves

$$
\Gamma_{B C}^{I} \operatorname{tr}\left(-\bar{\Psi}^{A} \gamma^{\mu} \Psi_{A} \bar{\Psi}^{B} \gamma_{\mu} \varepsilon^{I} X^{C}+\bar{\varepsilon}^{I} \gamma^{\mu} \Psi^{B} \bar{\Psi}_{A} \gamma_{\mu} \Psi^{A} X^{C}\right) .
$$

Inserting the identities above, so as to eliminate Dirac matrices while retaining the order of the matrices, which are implicitly multiplied, leaves

$$
\begin{aligned}
& \Gamma_{B C}^{I} \operatorname{tr}\left(2 \bar{\varepsilon}^{I} \Psi^{A} \bar{\Psi}_{A} \Psi^{B} X^{C}+\bar{\Psi}^{A} \Psi_{A} \bar{\varepsilon}^{I} \Psi^{B} X^{C}-2 \bar{\Psi}^{B} \Psi_{A} \bar{\varepsilon}^{I} \Psi^{A} X^{C}-\bar{\varepsilon}^{I} \Psi^{B} \bar{\Psi}_{A} \Psi^{A} X^{C}\right) \\
& \quad=i \operatorname{tr}\left(\bar{\Psi}^{A} \Psi_{A} \delta X_{B} X^{B}\right)-i \operatorname{tr}\left(\bar{\Psi}_{A} \Psi^{A} X^{B} \delta X_{B}\right)+2 \Gamma_{B C}^{I} \operatorname{tr}\left(\bar{\varepsilon}^{I} \Psi^{A}\left[\bar{\Psi}_{A} \Psi^{B} X^{C}-X^{C} \bar{\Psi}^{B} \Psi_{A}\right]\right) .
\end{aligned}
$$

Now consider varying the $X$ fields in the second term in $L_{4 a}$. This gives

$$
\begin{aligned}
-2 i \varepsilon_{A B C D} \operatorname{tr}\left(\bar{\Psi}^{A} \delta X^{B} \Psi^{C} X^{D}\right)= & -2 \tilde{\Gamma}^{I B E} \varepsilon_{A B C D} \operatorname{tr}\left(\bar{\Psi}^{A} \bar{\varepsilon}^{I} \Psi_{E} \Psi^{C} X^{D}\right) \\
= & -\varepsilon^{B E F G} \varepsilon_{A B C D} \Gamma_{F G}^{I} \operatorname{tr}\left(\bar{\Psi}^{A} \bar{\varepsilon}^{I} \Psi_{E} \Psi^{C} X^{D}\right) \\
= & \delta_{A C D}^{E F G} \Gamma_{F G}^{I} \operatorname{tr}\left(\bar{\Psi}^{A} \bar{\varepsilon}^{I} \Psi_{E} \Psi^{C} X^{D}\right) \\
= & -\delta_{A C D}^{E F G} \Gamma_{F G}^{I} \operatorname{tr}\left(\bar{\Psi}^{A} \Psi_{E} \bar{\varepsilon}^{I} \Psi^{C} X^{D}+\bar{\Psi}^{A} \varepsilon^{I} \bar{\Psi}_{E} \Psi^{C} X^{D}\right) \\
= & -2 i \operatorname{tr}\left(\bar{\Psi}^{A} \Psi_{A} \delta X_{B} X^{B}\right)+2 i \operatorname{tr}\left(\bar{\Psi}_{A} \Psi^{A} X^{B} \delta X_{B}\right) \\
& +2 i \operatorname{tr}\left(\bar{\Psi}^{A} \Psi_{B} \delta X_{A} X^{B}\right)-2 i \operatorname{tr}\left(\bar{\Psi}_{A} \Psi^{B} X^{A} \delta X_{B}\right) \\
& -2 \Gamma_{B C}^{I} \operatorname{tr}\left(\bar{\varepsilon}^{I} \Psi^{A}\left[\bar{\Psi}_{A} \Psi^{B} X^{C}-X^{C} \bar{\Psi}^{B} \Psi_{A}\right]\right)
\end{aligned}
$$

where we have used eq. (A.11). Here we have used the definition

$$
\delta_{A B C}^{D E F}=6 \delta_{A}^{[D} \delta_{B}^{E} \delta_{C}^{F]} .
$$

These two sets of terms combine to leave

$$
\begin{aligned}
-i \operatorname{tr}\left(\bar{\Psi}^{A} \Psi_{A} \delta X_{B} X^{B}\right)+i \operatorname{tr}\left(\bar{\Psi}_{A} \Psi^{A} X^{B} \delta X_{B}\right) \\
+2 i \operatorname{tr}\left(\bar{\Psi}^{B} \Psi_{A} \delta X_{B} X^{A}\right)-2 i \operatorname{tr}\left(\bar{\Psi}_{A} \Psi^{B} X^{A} \delta X_{B}\right) .
\end{aligned}
$$

These terms are canceled in turn by varying $X_{B}$ in $L_{4 \mathrm{~b}}$ and $L_{4 \mathrm{c}}$. Thus, terms of this structure in the supersymmetry transformations cancel for the choice of $L_{4}$ given in section 3. The adjoint terms cancel in the same way.

Since we now have the complete dependence of the action on spinor fields, we can deduce the spinor field equations of motion. They are

$$
\begin{aligned}
\gamma \cdot D \Psi^{A}= & -2 \varepsilon^{A B C D} X_{B} \Psi_{C} X_{D}-X_{B} X^{B} \Psi^{A}+\Psi^{A} X^{B} X_{B} \\
& -2 \Psi^{B} X^{A} X_{B}+2 X_{B} X^{A} \Psi^{B}
\end{aligned}
$$

and its adjoint

$$
\begin{aligned}
\gamma \cdot D \Psi_{A}= & 2 \varepsilon_{A B C D} X^{B} \Psi^{C} X^{D}+X^{B} X_{B} \Psi_{A}-\Psi_{A} X_{B} X^{B} \\
& +2 \Psi_{B} X_{A} X^{B}-2 X^{B} X_{A} \Psi_{B} .
\end{aligned}
$$


Determination of $\boldsymbol{\delta}_{\mathbf{3}} \boldsymbol{\Psi}$. Having determined $L_{4}$, we are now in a position to determine $\delta_{3} \Psi$ by computing terms of the schematic structure $\operatorname{tr}\left(\Psi_{A} D X_{B} X^{C} X_{D}\right)$, $\operatorname{tr}\left(\Psi_{A} X_{B} D X^{C} X_{D}\right)$, and $\operatorname{tr}\left(\Psi_{A} X_{B} X^{C} D X_{D}\right)$ that arise from varying the gauge fields in the $X$ kinetic term and varying the spinor fields in $L_{4}$. The adjoint terms work the same way. The terms of the indicated structure that arise from varying the gauge fields in the $X$ kinetic term are

$$
i \tilde{\Gamma}^{I B C} \operatorname{tr}\left[\bar{\Psi}_{B} \gamma^{\mu} \varepsilon^{I}\left(X_{C} X^{A} D_{\mu} X_{A}-D_{\mu} X_{A} X^{A} X_{C}+X_{A} D_{\mu} X^{A} X_{C}-X_{C} D_{\mu} X^{A} X_{A}\right)\right] .
$$

The terms of the indicated structure that arise from varying $L_{4 \mathrm{a}}$ are

$$
\begin{aligned}
& -2 i \varepsilon^{A B C D} \operatorname{tr}\left(\delta \bar{\Psi}_{D} X_{A} \Psi_{B} X_{C}\right)=-2 i \varepsilon^{A B C D} \Gamma_{D E}^{I} \operatorname{tr}\left(\bar{\Psi}_{B} \gamma^{\mu} \varepsilon^{I} X_{C} D_{\mu} X^{E} X_{A}\right) \\
& \left.=i \delta_{E F G}^{A B C} \tilde{\Gamma}^{I F G_{\operatorname{tr}}} \operatorname{t} \bar{\Psi}_{B} \gamma^{\mu} \varepsilon^{I} X_{C} D_{\mu} X^{E} X_{A}\right) \\
& =2 i \tilde{\Gamma}^{I B C} \operatorname{tr}\left(\bar{\Psi}_{B} \gamma^{\mu} \varepsilon^{I} X_{C} D_{\mu} X^{A} X_{A}+\bar{\Psi}_{C} \gamma^{\mu} \varepsilon^{I} X_{A} D_{\mu} X^{A} X_{B}\right. \\
& \left.+\bar{\Psi}_{A} \gamma^{\mu} \varepsilon^{I} X_{B} D_{\mu} X^{A} X_{C}\right) \text {. }
\end{aligned}
$$

The terms of the indicated structure that arise from varying $L_{4 \mathrm{~b}}$ are

$$
\begin{aligned}
i \operatorname{tr}\left(\delta \bar{\Psi}^{B} \Psi_{B} X_{A} X^{A}\right)-i \operatorname{tr}\left(\bar{\Psi}_{B} \delta \Psi^{B} X^{A} X_{A}\right) \\
\quad=i \tilde{\Gamma}^{I B C} \operatorname{tr}\left[\bar{\Psi}_{B} \gamma^{\mu} \varepsilon^{I}\left(D_{\mu} X_{C} X^{A} X_{A}-X_{A} X^{A} D_{\mu} X_{C}\right)\right]
\end{aligned}
$$

The terms of the indicated structure that arise from varying $L_{4 \mathrm{c}}$ are

$$
\begin{aligned}
& 2 i \operatorname{tr}\left(\bar{\Psi}_{A} \delta \Psi^{B} X^{A} X_{B}\right)-2 i \operatorname{tr}\left(\delta \bar{\Psi}^{B} \Psi_{A} X_{B} X^{A}\right) \\
& =2 i \tilde{\Gamma}^{I B C} \operatorname{tr}\left[\bar{\Psi}_{A} \gamma^{\mu} \varepsilon^{I}\left(X_{B} X^{A} D_{\mu} X_{C}+D_{\mu} X_{B} X^{A} X_{C}\right)\right]
\end{aligned}
$$

Adding these up, we obtain

$$
\begin{aligned}
& 2 i \tilde{\Gamma}^{I B C} \operatorname{tr}\left[\bar{\Psi}_{A} \gamma^{\mu} \varepsilon^{I} D_{\mu}\left(X_{B} X^{A} X_{C}\right)\right] \\
&+i \tilde{\Gamma}^{I B C} \operatorname{tr}\left[\bar{\Psi}_{B} \gamma^{\mu} \varepsilon^{I}\left(D_{\mu}\left(X_{C} X^{A} X_{A}\right)-D_{\mu}\left(X_{A} X^{A} X_{C}\right)\right)\right] .
\end{aligned}
$$

Thus, this can cancel against a variation of the spinor field in the spinor kinetic term for the choice

$$
\delta_{3} \Psi^{A}=\tilde{\Gamma}^{I A B} \varepsilon^{I}\left(X_{C} X^{C} X_{B}-X_{B} X^{C} X_{C}\right)-2 \tilde{\Gamma}^{I B C} \varepsilon^{I} X_{B} X^{A} X_{C}
$$

Determination of $\boldsymbol{V}=-\boldsymbol{L}_{\mathbf{6}}$. The next step is to determine $L_{6}$ by requiring that its $\delta X$ variation cancels against the $\delta_{3} \Psi$ variation of $L_{4}$. A key identity in the analysis is

$$
\Gamma_{A B}^{I} \tilde{\Gamma}^{I C D}=-2 \delta_{A B}^{C D}
$$

This is verified by showing that the two sides agree when contracted with $\delta_{C}^{B}$ as well as with $\left(\tilde{\Gamma}^{J} \Gamma^{K}-\tilde{\Gamma}^{K} \Gamma^{J}\right)^{B}$. Since these are 16 linearly independent $4 \times 4$ matrices, this constitutes a complete proof. 
The supersymmetry variation of $L_{4}$, keeping all terms containing $\Psi^{A}$ but not $\Psi_{A}$ (since the $\Psi_{A}$ terms work in the same way) is

$$
\begin{aligned}
\delta L_{4}= & -2 i \epsilon_{A B C D} \operatorname{tr}\left(\delta_{3} \bar{\Psi}^{A} X^{B} \Psi^{C} X^{D}\right) \\
& +i \operatorname{tr}\left(\delta_{3} \bar{\Psi}_{A}\left(X_{B} X^{B} \Psi^{A}-\Psi^{A} X^{B} X_{B}+2 \Psi^{B} X^{A} X_{B}-2 X_{B} X^{A} \Psi^{B}\right)\right),
\end{aligned}
$$

where, as derived previously,

$$
\begin{aligned}
& \delta_{3} \bar{\Psi}^{A}=\Gamma_{H K}^{I}\left[\frac{1}{2} \epsilon^{A C H K}\left(X_{D} X^{D} X_{C}-X_{C} X^{D} X_{D}\right)-\epsilon^{F G H K} X_{F} X^{A} X_{G}\right] \bar{\epsilon}^{I}, \\
& \delta_{3} \bar{\Psi}_{A}=\left[-\Gamma_{A C}^{I}\left(X^{C} X_{D} X^{D}-X^{D} X_{D} X^{C}\right)+2 \Gamma_{H K}^{I} X^{K} X_{A} X^{H}\right] \bar{\epsilon}^{I} .
\end{aligned}
$$

Expanding $\delta L_{4}$ is straightforward algebra and gives

$$
\begin{gathered}
\operatorname{tr}\left(3 X^{A} \delta X_{A} X^{B} X_{B} X^{C} X_{C}+3 \delta X_{A} X^{A} X_{B} X^{B} X_{C} X^{C}\right. \\
-2 X^{A} \delta X_{B} X^{B} X_{A} X^{C} X_{C}-2 X^{A} X_{B} X^{B} \delta X_{A} X^{C} X_{C}-2 X^{A} X_{B} X^{B} X_{A} X^{C} \delta X_{C} \\
+4 i \Gamma_{H K}^{I} \bar{\epsilon}^{I} \Psi^{A}\left[X^{H} X_{A} X^{B} X_{B} X^{K}+X^{B} X_{B} X^{H} X_{A} X^{K}+X^{H} X_{B} X^{K} X_{A} X^{B}\right. \\
\left.-X^{H} X_{B} X^{B} X_{A} X^{K}-X^{B} X_{A} X^{H} X_{B} X^{K}-X^{H} X_{A} X^{K} X_{B} X^{B}\right] \\
\left.+2 i \epsilon_{A B C D} \epsilon^{F G H} \Gamma_{H K}^{I} \bar{\epsilon}^{I} \Psi^{A} X^{B} X_{F} X^{C} X_{G} X^{D}\right) .
\end{gathered}
$$

The first two lines can be reproduced by varying

$$
V_{1}=\operatorname{tr}\left(X^{A} X_{A} X^{B} X_{B} X^{C} X_{C}+X_{A} X^{A} X_{B} X^{B} X_{C} X^{C}-2 X^{A} X_{B} X^{B} X_{A} X^{C} X_{C}\right) .
$$

The last line cancels the third and fourth lines and contributes additional terms to $V_{1}$, as we will now show. For this purpose, the following identity is useful:

$$
\begin{aligned}
2 \epsilon_{A B C D} \epsilon^{F G H K} \Gamma_{H K}^{I} & =\epsilon_{L B C D} \epsilon^{F G H K} \Gamma_{H K}^{J}\left(2 \delta^{I J} \delta_{A}^{L}\right) \\
& =\epsilon_{L B C D} \epsilon^{F G H K} \Gamma_{H K}^{J}\left(\Gamma_{A M}^{I} \tilde{\Gamma}^{J M L}+\Gamma_{A M}^{J} \tilde{\Gamma}^{I M L}\right) \\
& =4 \delta_{B C D}^{F G M} \Gamma_{A M}^{I}+2\left(\delta_{B C D}^{G P Q} \delta_{A}^{F}-\delta_{B C D}^{F P Q} \delta_{A}^{G}\right) \Gamma_{P Q}^{I}
\end{aligned}
$$

where we have used (A.19) to go from the second line to the third line. Plugging this identity into the last line of (A.2Q) gives

$$
\begin{aligned}
\operatorname{tr}(- & 4 \delta_{B C D}^{F G M} \delta X_{M} X^{B} X_{F} X^{C} X_{G} X^{D} \\
& \left.+2 i \Gamma_{H K}^{I} \bar{\epsilon}^{I} \Psi^{A}\left(\delta_{B C D}^{G H K} \delta_{A}^{F}-\delta_{B C D}^{F H K} \delta_{A}^{G}\right) X^{B} X_{F} X^{C} X_{G} X^{D}\right) .
\end{aligned}
$$

Expanding the first term in (A.21) gives

$$
\begin{aligned}
4 \operatorname{tr}[ & -X^{D} \delta X_{D} X^{F} X_{F} X^{G} X_{G}-\delta X_{B} X^{B} X_{C} X^{C} X_{D} X^{D}-\delta X_{C} X^{G} X_{D} X^{C} X_{G} X^{D} \\
& \left.+\delta X_{C} X^{F} X_{F} X^{C} X_{D} X^{D}+\delta X_{B} X^{B} X_{D} X^{G} X_{G} X^{D}+\delta X_{D} X^{G} X_{C} X^{C} X_{G} X^{D}\right]
\end{aligned}
$$


which also comes from varying

$$
\begin{aligned}
V_{2}=\operatorname{tr}\left(-\frac{4}{3} X^{A} X_{A} X^{B} X_{B} X^{C} X_{C}-\frac{4}{3} X_{A} X^{A} X_{B} X^{B} X_{C} X^{C}\right. \\
\left.-\frac{4}{3} X_{A} X^{B} X_{C} X^{A} X_{B} X^{C}+4 X^{A} X_{B} X^{B} X_{A} X^{C} X_{C}\right) .
\end{aligned}
$$

Adding this potential to eq. (A.20) gives the total potential

$$
\begin{aligned}
V=-\frac{1}{3} \operatorname{tr}\left[X^{A} X_{A} X^{B} X_{B} X^{C} X_{C}+X_{A} X^{A} X_{B} X^{B} X_{C} X^{C}\right. \\
\left.+4 X_{A} X^{B} X_{C} X^{A} X_{B} X^{C}-6 X^{A} X_{B} X^{B} X_{A} X^{C} X_{C}\right] .
\end{aligned}
$$

Furthermore, straightforward algebra shows that the second term in eq. (A.21) precisely cancels the terms in the third and fourth lines of eq. (A.20). So we conclude that the variation of $L_{4}$ is completely canceled by varying $-V$. This expression agrees with the potential obtained in [1, 2].

It is also interesting to note that $V$ is proportional to the trace of the absolute square of the $X^{3}$ expression that appears in $\delta_{3} \Psi$. Specifically,

$$
V=\frac{1}{6} \operatorname{tr}\left(N^{I A} N_{A}^{I}\right)
$$

which is straightforward to verify using eq. (A.19).

Conserved supersymmetry current. The conserved supersymmetry current of the $\mathrm{U}(N) \times \mathrm{U}(N)$ theory, generalizing the expression given earlier for the $\mathrm{U}(1) \times \mathrm{U}(1)$ theory, is

$$
Q_{\mu}^{I}=\operatorname{tr}\left(M_{A}^{I} \gamma_{\mu} \Psi^{A}\right)+\operatorname{tr}\left(M^{I A} \gamma_{\mu} \Psi_{A}\right) .
$$

Here

$$
M_{A}^{I}=-\Gamma_{A B}^{I} \gamma \cdot D X^{B}+N_{A}^{I}
$$

and

$$
M^{I A}=\tilde{\Gamma}^{I A B} \gamma \cdot D X_{B}+N^{I A}
$$

are quantities that appear in the supersymmetry variations of the spinor fields $\bar{\Psi}_{A}$ and $\bar{\Psi}^{A}$, respectively. The quantity $N_{A}^{I}$ and its adjoint $N^{I A}$ were defined in eqs. (3.20) and (3.21). The verification that this current is conserved as a consequence of the equations of motion is rather tedious. In any case, it would be redundant, since it is equivalent to the verification of the supersymmetry of the action, which we have just carried out.

Conformal supersymmetry. In the $\mathrm{U}(1) \times \mathrm{U}(1)$ case, we found that the conformal supersymmetries can be described by replacing $\varepsilon^{I}$ in the Poincaré supersymmetries by $\gamma \cdot x \eta^{I}$ and by adding an additional term to the spinor field transformations

$$
\delta^{\prime} \Psi_{A}=\Gamma_{A B}^{I} X^{B} \eta^{I}
$$

and its adjoint. Let us now verify that the same rule continues to work for $N>1$. Most terms cancel as a consequence of the Poincaré supersymmetry. The remaining ones that 
need to cancel separately are those that arise from the derivative in $i \bar{\Psi}_{A} \gamma \cdot D \delta \Psi^{A}$ acting on the explicit $x^{\mu}$ in the $\eta^{I}$ transformation. This gives

$$
i \bar{\Psi}_{A}\left[\tilde{\Gamma}^{I A B}\left(\gamma \cdot D X_{B}+3 X_{C} X^{C} X_{B}-3 X_{B} X^{C} X_{C}\right)-6 \tilde{\Gamma}^{I B C} X_{B} X^{A} X_{C}\right] \eta^{I} .
$$

The first term in this expression is canceled by the $\delta^{\prime} \Psi^{A}$ variation of the spinor kinetic term. The remaining terms need to cancel against the $\delta^{\prime} \Psi$ variation of $L_{4}$. The relevant terms that arise in this way are

$$
\begin{aligned}
& 2 i \varepsilon^{A B C D} \operatorname{tr}\left(\delta^{\prime} \bar{\Psi}_{A} X_{B} \Psi_{C} X_{D}\right)+i \operatorname{tr}\left(\delta^{\prime} \bar{\Psi}^{A} \Psi_{A} X_{B} X^{B}\right)-i \operatorname{tr}\left(\bar{\Psi}_{A} \delta^{\prime} \Psi^{A} X^{B} X_{B}\right) \\
& 2 i \operatorname{tr}\left(\bar{\Psi}_{A} \delta^{\prime} \Psi^{B} X^{A} X_{B}\right)-2 i \operatorname{tr}\left(\delta^{\prime} \bar{\Psi}^{B} \Psi_{A} X_{B} X^{A}\right) .
\end{aligned}
$$

By manipulations similar to those described previously, the first term in this expression can be recast in the form

$$
2 i \tilde{\Gamma}^{I B C} \operatorname{tr}\left(\bar{\Psi}_{A} X_{B} X^{A} X_{C}+\bar{\Psi}_{B} X_{C} X^{A} X_{A}+\bar{\Psi}_{C} X_{A} X^{A} X_{B}\right) \eta^{I}
$$

Combining this with the other four terms leaves

$$
i \bar{\Psi}_{A}\left[\tilde{\Gamma}^{I A B}\left(-3 X_{C} X^{C} X_{B}+3 X_{B} X^{C} X_{C}\right)+6 \tilde{\Gamma}^{I B C} X_{B} X^{A} X_{C}\right] \eta^{I} .
$$

This provides the desired cancellation, which proves that the theory has conformal supersymmetry.

Taken together with the $\mathcal{N}=6$ Poincaré supersymmetry, the conformal supersymmetry implies that the theory has the full $O S p(6 \mid 4)$ superconformal symmetry. Even though this result is necessary for a dual AdS interpretation, it was not at all obvious that this symmetry would hold. After all, it is not a logical consequence of the other symmetries that have been verified.

Accordingly, the conserved conformal supersymmetry currents in the $\mathrm{U}(N) \times \mathrm{U}(N)$ theory are given by

$$
S_{\mu}^{I}=\gamma \cdot x Q_{\mu}^{I}-\Gamma_{A B}^{I} \operatorname{tr}\left(X^{B} \gamma_{\mu} \Psi^{A}\right)+\tilde{\Gamma}^{I A B} \operatorname{tr}\left(X_{B} \gamma_{\mu} \Psi_{A}\right)
$$

As a check on our analysis, let us compute the divergence. The $D X^{B}$ terms cancel leaving

$$
\partial^{\mu} S_{\mu}^{I}=\operatorname{tr}\left(3 N_{A}^{I} \Psi^{A}+3 N^{I A} \Psi_{A}-\Gamma_{A B}^{I} X^{B} \gamma \cdot D \Psi^{A}+\tilde{\Gamma}^{I A B} X_{B} \gamma \cdot D \Psi_{A}\right)
$$

where $N_{A}^{I}$ and $N^{I A}$ are as before. Using the spinor field equations of motion (A.15) and (A.16) to eliminate $\gamma \cdot D \Psi^{A}$ and $\gamma \cdot D \Psi_{A}$, the terms in $\partial^{\mu} S_{\mu}^{I}$ that involve $\Psi^{A}$ are

$$
\begin{aligned}
3 \operatorname{tr}\left(N_{A}^{I} \Psi^{A}\right) & +2 \varepsilon_{A C D E} \tilde{\Gamma}^{I A B} \operatorname{tr}\left(X_{B} X^{C} \Psi^{D} X^{E}\right) \\
& -\Gamma_{A B}^{I} \operatorname{tr}\left(X^{B}\left[-X_{C} X^{C} \Psi^{A}+\Psi^{A} X^{C} X_{C}-2 \Psi^{C} X^{A} X_{C}+2 X_{C} X^{A} \Psi^{C}\right]\right) .
\end{aligned}
$$

A short calculation, similar to previous ones, shows that this vanishes. 


\section{References}

[1] O. Aharony, O. Bergman, D.L. Jafferis and J. Maldacena, $N=6$ superconformal Chern-Simons-matter theories, M2-branes and their gravity duals, arXiv:0806.1218.

[2] M. Benna, I. Klebanov, T. Klose and M. Smedback, Superconformal Chern-Simons theories and $A d S_{4} / C F T_{3}$ correspondence, arXiv:0806.1519.

[3] J. Bhattacharya and S. Minwalla, Superconformal indices for $\mathcal{N}=6$ Chern Simons theories, arXiv:0806.3251.

[4] T. Nishioka and T. Takayanagi, On type IIA Penrose limit and $N=6$ Chern-Simons theories, JHEP 08 (2008) 001 arXiv:0806.3391.

[5] Y. Honma, S. Iso, Y. Sumitomo and S. Zhang, Scaling limit of $N=6$ superconformal Chern-Simons theories and Lorentzian Bagger-Lambert theories, arXiv:0806.3498.

[6] Y. Imamura and K. Kimura, Coulomb branch of generalized ABJM models, arXiv:0806.3727.

[7] J.A. Minahan and K. Zarembo, The Bethe ansatz for superconformal Chern-Simons, arXiv:0806.3951.

[8] A. Armoni and A. Naqvi, A non-supersymmetric large-N $3 D$ CFT and its gravity dual, arXiv:0806.4068.

[9] D. Gaiotto, S. Giombi and X. Yin, Spin chains in $N=6$ superconformal Chern-Simons-matter theory, arXiv:0806.4589.

[10] G. Grignani, T. Harmark and M. Orselli, The $\mathrm{SU}(2) \times \mathrm{SU}(2)$ sector in the string dual of $N=6$ superconformal Chern-Simons theory, arXiv:0806.4959.

[11] K. Hosomichi, K.-M. Lee, S. Lee, S. Lee and J. Park, $N=5,6$ superconformal Chern-Simons theories and M2-branes on orbifolds, arXiv:0806.4977.

[12] A. Hanany, N. Mekareeya and A. Zaffaroni, Partition functions for membrane theories, arXiv:0806.4212.

[13] J. Bagger and N. Lambert, Three-algebras and $N=6$ Chern-Simons gauge theories, arXiv:0807.0163.

[14] S. Terashima, On M5-branes in $N=6$ membrane action, arXiv:0807.0197.

[15] G. Grignani, T. Harmark, M. Orselli and G.W. Semenoff, Finite size giant magnons in the string dual of $N=6$ superconformal Chern-Simons theory, arXiv:0807.0205.

[16] J.H. Schwarz, Superconformal Chern-Simons theories, JHEP 11 (2004) 078 hep-th/0411077.

[17] A. Basu and J.A. Harvey, The M2-M5 brane system and a generalized Nahm's equation, Nucl. Phys. B 713 (2005) 136 hep-th/0412310.

[18] J. Bagger and N. Lambert, Modeling multiple M2's, Phys. Rev. D 75 (2007) 045020 hep-th/0611108.

[19] J. Bagger and N. Lambert, Gauge symmetry and supersymmetry of multiple M2-branes, Phys. Rev. D 77 (2008) 065008 arXiv:0711.0955.

[20] J. Bagger and N. Lambert, Comments on multiple M2-branes, JHEP 02 (2008) 105 arXiv:0712.3738. 
[21] A. Gustavsson, Algebraic structures on parallel M2-branes, arXiv:0709.1260.

[22] A. Gustavsson, Selfdual strings and loop space Nahm equations, JHEP 04 (2008) 083 arXiv:0802.3456.

[23] M.A. Bandres, A.E. Lipstein and J.H. Schwarz, $N=8$ superconformal Chern-Simons theories, JHEP 05 (2008) 025 arXiv:0803.3242.

[24] M. Van Raamsdonk, Comments on the Bagger-Lambert theory and multiple M2-branes, JHEP 05 (2008) 105 arXiv: 0803.3803 .

[25] G. Papadopoulos, M2-branes, 3-Lie algebras and Plücker relations, JHEP 05 (2008) 054 arXiv:0804.2662.

[26] J.P. Gauntlett and J.B. Gutowski, Constraining maximally supersymmetric membrane actions, arXiv:0804.3078.

[27] J. Gomis, G. Milanesi and J.G. Russo, Bagger-Lambert theory for general Lie algebras, JHEP 06 (2008) 075 arXiv:0805.1012.

[28] S. Benvenuti, D. Rodriguez-Gomez, E. Tonni and H. Verlinde, $N=8$ superconformal gauge theories and M2 branes, arXiv:0805.1087.

[29] P.-M. Ho, Y. Imamura and Y. Matsuo, M2 to D2 revisited, JHEP 07 (2008) 003 arXiv:0805.1202.

[30] M.A. Bandres, A.E. Lipstein and J.H. Schwarz, Ghost-free superconformal action for multiple M2-branes, JHEP 07 (2008) 117 arXiv:0806.0054.

[31] J. Gomis, D. Rodriguez-Gomez, M. Van Raamsdonk and H. Verlinde, Supersymmetric Yang-Mills theory from lorentzian three-algebras, arXiv:0806.0738.

[32] B. Ezhuthachan, S. Mukhi and C. Papageorgakis, D2 to D2, JHEP 07 (2008) 041 arXiv:0806.1639.

[33] D. Gaiotto and E. Witten, Janus configurations, Chern-Simons couplings, and the $\theta$-angle in $N=4$ super Yang-Mills theory, arXiv:0804.2907.

[34] K. Hosomichi, K.-M. Lee, S. Lee, S. Lee and J. Park, $N=4$ superconformal Chern-Simons theories with hyper and twisted hyper multiplets, JHEP 07 (2008) 091 arXiv:0805.3662].

[35] A.S. Cattaneo, P. Cotta-Ramusino, J. Fröhlich and M. Martellini, Topological BF theories in three-dimensions and four-dimensions, J. Math. Phys. 36 (1995) 6137 hep-th/9505027.

[36] N. Lambert and D. Tong, Membranes on an orbifold, arXiv:0804.1114.

[37] J. Distler, S. Mukhi, C. Papageorgakis and M. Van Raamsdonk, M2-branes on M-folds, JHEP 05 (2008) 038 arXiv:0804.1256. 